\title{
Autonomic Denervation of Lymphoid Organs Leads to Epigenetic Immune Atrophy in a Mouse Model of Krabbe Disease
}

\author{
Francesca Galbiati, ${ }^{1}$ Veronica Basso, ${ }^{5}$ Ludovico Cantuti, ${ }^{1}$ Maria Irene Givogri, ${ }^{1}$ Aurora Lopez-Rosas, ${ }^{1}$ Nicolas Perez, ${ }^{2}$ \\ Chenthamarakshan Vasu, ${ }^{3}$ Hongmei Cao,${ }^{4}$ Richard van Breemen, ${ }^{4}$ Anna Mondino, ${ }^{5}$ and Ernesto R. Bongarzone ${ }^{1}$ \\ Departments of ${ }^{1}$ Anatomy and Cell Biology, ${ }^{2}$ Microbiology and Immunology, and ${ }^{3}$ Surgery, College of Medicine, and ${ }^{4}$ Medicinal Chemistry and \\ Pharmacognosy, College of Pharmacy, University of Illinois at Chicago, Chicago, Illinois 60612-7308, and ${ }^{5}$ Lymphocyte Activation Unit, San Raffaele \\ Scientific Institute, Milan 20132, Italy
}

Lysosomal $\beta$-galactosylceramidase deficiency results in demyelination and inflammation in the nervous system causing the neurological Krabbe disease. In the Twitcher mouse model of this disease, we found that neurological symptoms parallel progressive and severe lymphopenia. Although lymphopoiesis is normal before disease onset, primary and secondary lymphoid organs progressively degenerate afterward. This occurs despite preserved erythropoiesis and leads to severe peripheral lymphopenia caused by reduced numbers of T cell precursors and mature lymphocytes. Hematopoietic cell replacement experiments support the existence of an epigenetic factor in mutant mice reconcilable with a progressive loss of autonomic axons that hampers thymic functionality. We propose that degeneration of autonomic nerves leads to the irreversible thymic atrophy and loss of immune-competence. Our study describes a new aspect of Krabbe disease, placing patients at risk of immune-related pathologies, and identifies a novel target for therapeutic interventions.

Key words: thymus; Krabbe disease; autonomic system; psychosine; T cells; inflammation; hematopoietic stem cells; axonal degeneration

\section{Introduction}

Krabbe disease or globoid cell leukodystrophy (GLD) is characterized by progressive demyelination of the nervous system and the formation of multinucleated macrophages cells (globoid cells) (Bullard and Southard, 1906; Suzuki, 2003), followed by early death. Deficiency of $\beta$-galactosylceramidase (GALC) causes the cellular storage of galactosylsphingosine (psychosine) and lactosylceramide in GLD (Wenger et al., 2001).

GLD is more frequent in infants than in adults (Hagberg et al., 1970; Crome et al., 1973), and it has remained without a definitive long-term cure. Gene therapy, enzyme replacement, and cell therapy have been tested in animal models of the disease as well as in patients with varied results (Umezawa et al., 1985; Hoogerbrugge et al., 1989; Krivit et al., 1995; Wu et al., 2000, 2001; Luddi et al., 2001; Luzi et al., 2005; Yagi et al., 2004; Dolcetta et al., 2006). Recently, transplantation of hematopoietic stem cells (HSCs) in asymptomatic or presymptomatic neonates and infants has become the preferred therapy in many cases of Krabbe

Received July 26, 2007; revised 0ct. 22, 2007; accepted 0ct. 24, 2007.

This work was supported by the San Raffaele Scientific Institute (A.M.) and by a University of Illinois at Chicago intramural seed grant (E.R.B.). We thank the anonymous reviewers for their excellent inputs. E.R.B. dedicates this paper to Eduardo Soto, Giannina Pasquini, and Tony Campagnoni, mentors and friends.

Correspondence should be addressed to either of the following: Dr. Anna Mondino, Lymphocyte Activation Unit, Cancer Immunotherapy and Gene Therapy Program, San Raffaele Scientific Institute, Via Olgettina 58, Milano 20132, Italy, E-mail:mondino.anna@hsr.it; or Dr. Ernesto R. Bongarzone, Department of Anatomy and Cell Biology, College of Medicine, University of Illinois at Chicago, 808 South Wood Street, M/C 512, Chicago, IL 60612-7308, E-mail: ebongarz@uic.edu.

DOI:10.1523/JNEUROSCI.3379-07.2007

Copyright $\odot 2007$ Society for Neuroscience $\quad 0270-6474 / 07 / 2713730-09 \$ 15.00 / 0$
(Krivit et al., 1995; Krivit et al., 1998; Escolar et al., 2005). Of note, studies of hematopoietic replacement in Twitcher mice have shown that whereas this therapy significantly prolongs survival and improves symptomology, it does not cure them. The reason for this limitation remains unknown.

Of central relevance for the success of hematopoietic cell therapies, the immune-competence of the host has to be adequate to allow full engraftment of donor cells and the subsequent ability to generate all immune-cell types. In the case of Krabbe disease, whether GALC deficiency and/or psychosine accumulation exert direct effect on the physiology of the immune system remains to be defined. In this study, we addressed this question by examining the functionality of the Twitcher immune system before disease onset and during disease progression.

\section{Materials and Methods}

Animals. For the Twitcher colony, breeder Twitcher heterozygous mice (C57BL/6J, twi/+; Jackson Laboratories, Bar Harbor, ME) were maintained under standard housing conditions with approval of the Animal Care and Use Committee. Treated and untreated mice were allowed to survive as long as humanely possible. When a mouse reached a moribund condition, it was killed.For the CD45.1 colony, C57BL/6J congenic mice carrying the CD45.1 allele were kept under standard housing conditions and used either as marrow donors for Twitcher transplant or as recipients for Twitcher bone marrow. Where indicated, mice were tail-bled, and the cells were analyzed by flow cytometry. DNA was extracted from clipped tails of newborn mice at postnatal day 1 (P1) to P2 and used for PCR genotyping as described previously (Sakai et al., 1996; Dolcetta et al., 2006).

Irradiation and bone marrow transplant. Twitcher, their wild-type lit- 
termates, and C57BL/6J CD45.1 mice (5 d after birth) were subjected to a sublethal whole body irradiation from a ${ }^{137} \mathrm{Cs}$ source of gamma rays using a Gilardoni Rad Gil Irradiator. Mice were put in a wheel and kept in a prone position during irradiation treatment. The animals received a total dose of 6.2 gray by two cycles of $4.1 \mathrm{~min} 200 \mathrm{~kW}$ (3.1 gray each). Donor mice, C57BL/6J CD45.1 (8-12 weeks of age), and Twitcher mice ( $26 \mathrm{~d}$ of age) were killed and bone marrow cells were obtained by flushing tibiae and femora with $0.9 \%$ saline using a $10 \mathrm{ml}$ syringe ( $30 \mathrm{G}$ needles). Cells were then filtered with a $44 \mu \mathrm{m}$ cell strainer (BD Bioscience, San Diego, CA) to remove debris and centrifuged at $1500 \mathrm{rpm}$ for $5 \mathrm{~min}$. The pellet was incubated for $10 \mathrm{~min}$ at $4^{\circ} \mathrm{C}$ with lysing buffer $(155 \mathrm{~mm}$ ammonium chloride, $10 \mathrm{~mm}$ potassium bicarbonate, $0.1 \mathrm{~mm}$ EDTA, $\mathrm{pH} 8$ ) to eliminate red blood cells. Bone marrow cells were counted, and $10 \times$ $10^{6}$ cells were resuspended in $50 \mu \mathrm{l}$ of saline and injected intraperitoneally in 6-d-old recipient mice. Treated mice were returned in the original cage with the mothers, and no additional supportive care was given.

White and red blood cell count. Two hundred microliters of whole blood, obtained by tail-bleeding of the mice, were collected in tubes containing $40 \mu \mathrm{l}$ of EDTA $(45 \mathrm{mg} / \mathrm{ml})$. Blood samples were processed for counts using a Sysmez KX-21N hemocytometer.

Cell death in vitro analysis. The apoptotic effect of psychosine on mouse thymocytes and splenocytes was tested using mitochondrialspecific dye Tetramethyl-rhodamineethylester (TMRE). Splenocytes or thymocytes were plated in a 96-well, U-bottom tissue culture plate $(2 \times$ $10^{5}$ cells/well) in complete medium consisting of Roswell Park Memorial Institute 1640 supplemented with $2 \mathrm{~mm}$ L-glutamine, $15 \mathrm{~mm}$ HEPES, 1 mu sodium pyruvate, $5 \times 10.5$ м 2-mercaptho-ethanol, $100 \mathrm{U} / \mathrm{ml}$ penicillin, $0.1 \mathrm{mg} / \mathrm{ml}$ streptomycin, $1 \mu \mathrm{g} / \mathrm{ml}$ fungizone, and $10 \% \mathrm{FBS}$. Various concentrations of psychosine ( $200 \mathrm{~nm}$ to $25 \mu \mathrm{M}$ ) were added to wells in triplicate and incubated for $24 \mathrm{~h}$ at $37^{\circ} \mathrm{C}$. After incubation, cells were washed once using complete medium and incubated in $2.5 \mathrm{~nm}$ final TMRE solution in $10 \mathrm{mM} \mathrm{HEPES}$ for $30 \mathrm{~min}$ at $37^{\circ} \mathrm{C}$. Cells were washed twice and resuspended in PBS containing $0.5 \%$ BSA. Stained cells were analyzed using FACScalibur (Becton Dickinson, Mountain View, CA) and then analyzed using CellQuest software.

Flow cytometry analysis. Mice were killed at P15, P30, P40, and P80. Blood, peripheral lymph nodes (LNs), spleen, thymus, and bone marrow were collected by intracardiac bleeding or surgical resection. LNs, spleen, thymus, and bone marrow were homogenized into single-cell suspensions, and blood samples were depleted of red blood cells by cell lyses (Lonza, Walkersville, MD). Viable cells were enumerated by Trypan Blue-assisted counts. The cells were then incubated with a blocking buffer (5\% rat serum and 95\% culture supernatant of 2.4G2 anti-FcR monoclonal antibody ( $\mathrm{mAb}$ )-producing hybridoma cells) for $20 \mathrm{~min}$ at room temperature to saturate the FcRs. Cells from LNs, spleen, and blood cells were stained for CD4, CD8, B220, and CD11b. Thymocytes subpopulations were detected by staining with anti-CD4, anti-CD8, anti$\mathrm{CD} 25$, and anti-CD44 mAbs. Bone marrow precursor cells were identified by staining for CD11b, Ter 119, SCA-1, and GR-1 (all from BD PharMingen, San Diego, CA). Samples were collected at FACScalibur (Becton Dickinson) and then analyzed using CellQuest software.

Gene expression analysis. RNA was extracted with TRIZOL and retrotranscribed with cDNA synthesis Superscript (Invitrogen, San Diego, CA). Real-time PCR analysis was performed on a Bio-Rad iCycler4 with the Bio-Rad Sybr Green Supermix. The relative comparison between the samples was done with the $\Delta \Delta C t$ method using glyceraldehyde-3phosphate dehydrogenase gene (GAPDH) as a housekeeping gene. The primers were designed with the Beacon Designer software (Bio-Rad) and the Perlprimer software (Livak and Schmittgen, 2001), tested on Blast to avoid nonspecific binding. Serial dilutions of the cDNA were used to calculate efficiency and correlation coefficient. Primers were used only when equal at least to $93 \%$ and 0.991 , respectively). The following primers were used: $\beta 1$-adrenergic receptor, forward 5-TCGGCGTTGG TGTCCTTCC, reverse 5-TGACGAAATCGCAGCACTTGG; $\beta 2$ adrenergic receptor, forward 5-CGTCAACTCTGCCTTCAATCCTC, reverse 5-GTAGTCCGTTCTGCCGTTGC; and GAPDH, forward 5-TGCCACTCAGAAGACTGTGG, reverse 5-TTCAGCTCTGG GATGACCTT.
Immunohistology procedures. Tissue was cryosectioned and processed for diamino-benzidine-light and confocal fluorescence immunocytochemistry as described previously (Givogri et al., 2006). The antibodies used were the following: anti-CD4, CD8, and CD11c (BD PharMingen), anti-tyrosine hydroxylase (TH; Chemicon, Temecula, CA), and anticholine acetyl-transferase (ChaT; Chemicon). Hematoxylin staining was performed on cryosections with Hematoxylin QS (Vector Laboratories, Burlingame, CA). Sections were observed through an Olympus Optical (Tokyo, Japan) confocal microscope and in a Zeiss (Oberkochen, Germany) upright microscope with Nomarski optics. To quantify $\mathrm{TH}+\mathrm{fi}-$ bers in the thymic cortex, 10 serial sections (20 $\mu \mathrm{m}$ each) per thymus were stained as described above. The number of $\mathrm{TH}+$ nerve endings were counted in $0.1 \mathrm{~mm}^{2}$ area (three areas per section). Counting was performed in two thymuses per genotype at P20 and P30.

GALC activity and mass spectrometry determination of psychosine. Tissue samples were weighed, homogenized, and processed for GALC enzyme activity as described previously (Dolcetta et al., 2006). Samples were also extracted for psychosine. In brief, samples were extracted using chloroform and methanol and then partially purified on a strong cation exchanger column. After evaporation to dryness, each residue was dissolved in $200 \mu \mathrm{l}$ of methanol containing $5 \mathrm{~mm}$ ammonium formate, and $10 \mu \mathrm{l}$ aliquots were analyzed using liquid chromatography tanden mass spectrometry (LC-MS/MS). The HPLC system consisted of Shimadzu (Columbia, MD) LC-10Advp pumps with a Leap (Carrboro, NC) HTS PAL autosampler. Psychosine was measured using a Waters XTerra 3.5 $\mu \mathrm{m}, \mathrm{MS} \mathrm{C}_{18}, 2.1 \times 100 \mathrm{~mm}$ analytical column. Positive ion electrospray tandem mass spectrometry was performed using an Applied Biosystems (Foster City, CA) API 4000 triple quadrupole mass spectrometer with a collision energy of $29 \mathrm{eV}$ for psychosine and $37 \mathrm{eV}$ for the internal standard, lyso-lactosylceramide. The dwell time was 1.0 s/ion during multiple reaction monitoring.

Electron microscopy. Thymuses were collected from P40 mice and then fixed with $5 \%$ glutaraldehyde. Samples were processed for electron microscopy as described previously (Givogri et al., 2002) embedded in Araldite, and ultrathin sections $(60 \mathrm{~nm})$ were collected on Formvarcoated one-hole grids and stained with osmium tetroxide and lead acetate. Samples were observed in a JEOL JEM 100cx II electron microscope.

Statistical analysis. Results are the average from three to five different experiments and are expressed as mean \pm SE (SE). Data were analyzed by the Student's $t$ test. $p$ values $<0.05$ were considered statistically significant.

\section{Results}

\section{Progressive loss of mononuclear cells in peripheral blood of Twitcher mutants}

The Twitcher mouse is a natural model for infantile GLD. Twitcher mice are born normal, and until P15 are undistinguishable from control littermates. Thereafter, Twitcher mutants show progressive inability to gain weight (Fig. 1A). Evidence of neurological disease first appears at approximately day 20 when the mice show progressive tremor and ataxia, culminating with death at approximately P42 (Fig. $1 B$ ). Several therapeutic strategies are based on the use of HSC transplant. Despite this, no data are available on the state of immune-competence of the Twitcher mutant. To examine this, white blood cell (WBC) and red blood cell (RBC) numbers were independently measured before and after disease onset. Hematopoiesis appeared normal during early life (P15), but not at later times, when the number of WBCs progressively decreased to reach $9.2 \%$ of that of control mice at the time of death (Fig. 1C). In contrast, the numbers of RBCs were similar in Twitcher and wild-type mice at all ages considered (Fig. 1D).

\section{GLD mice develop a progressive, age-related lymphoid organ failure}

To analyze the reasons for reduced WBC counts in Twitcher mutants, primary and secondary lymphoid organs were analyzed. Al- 
though at P15 thymus, LNs and spleen of Twitcher and wild-type mice appeared comparable (Fig. 2A), and mutant organs were smaller at later ages (Fig. $2 A$ ). Total cell counts per organ (cellularity) confirmed the age-related alteration. Thymus, spleen, and LN cellularity were normal in mutant mice up to P15, but then they decreased by P30 and $\mathrm{P} 40$ (Fig. 2C-E). Bone marrow (isolated from tibiae and femurs) and peripheral blood cellularity was reduced primarily toward the end of Twitcher life (Fig. 2B,F).

Maturation of primary and secondary lymphoid organs in GLD mice

Subsets representation in Twitcher primary and secondary lymphoid organs was examined by flow cytometric analysis using lineage-specific markers during disease progression. The relative number of CD4/ CD8 double negative (DN), CD4/CD8 double positive (DP), and CD4 or CD8 single positive (SP) thymocyte subsets increased between P15 and P40 in wild-type thymuses (Fig. $3 A-D$ ). In contrast, whereas numbers of DN, DP, and SP thymocytes were comparable in Twitcher and control littermates at P15, they progressively decreased in mutant mice (Fig. 3A$D)$. Thymus development appeared to be hampered at the earliest stages, as shown by the decreased number of DN subsets (identified by the relative expression of CD44 and CD25) (data not shown). Thymic involution correlated with peripheral lymphopenia, evidenced by reduced lymphocyte counts in the spleen (Table 1) and lymph nodes (data not shown). In the bone marrow, the number of B220 + B-lymphocytes in Twitcher mice was 56 and $17 \%$ at P30 and P40, respectively (Table 1). In contrast, SCA- ${ }^{+}$hematopoietic stem cells and GR-1 ${ }^{+}$ granulocytes were not statistically different in mutant bone marrow at P30 but were reduced to 31 and $34 \%$ of control values at P40. In contrast, the number of Ter $119^{+}$erythroid progenitors remained normal at all time points (Table 1). These data indicate that disease progression in Twitcher mutants is paralleled by a progressive loss of immune-competence, hampering the generation of lymphocytes but sparing that of erythroid cells.

\section{Atrophy of GLD thymus comprises caspase-3-mediated apoptosis and increased intracellular vesicle abnormalities} To better understand the progressive and nonselective reduction in thymic subsets, we analyzed thymic cell death by caspase- 3 detection. Figure 4, $A$ and $E$, shows that apoptotic cells (red fluorescence for caspase 3 active fragment) were more frequently found in P15 Twitcher cortex (Fig. 4A) and medulla (Fig. 4E) than in the corresponding regions of the wild-type thymuses (Fig. $4 B, F$ ). Double labeling using anti-CD4 (Fig. $4 A, B, E, F$ ), anti-CD8 (data not shown), and anti-CD11c (Fig. 4C, D, G,H) showed caspase 3 in $\mathrm{CD}^{+}$(Fig. $\left.4 A, E\right), \mathrm{CD}^{+}$(data not shown), and CD11c ${ }^{+}$(Fig. $4 C, G$ ) Twitcher cells. Comparable results were obtained at P40 (data not shown).

Microscopy examination of the atrophic Twitcher thymus revealed abundant abnormalities in thymic cells. Hematoxylin staining showed that the thymic cortex was progressively reduced and almost absent in Twitcher thymus by $\mathrm{P} 40$ (data not shown). Electron
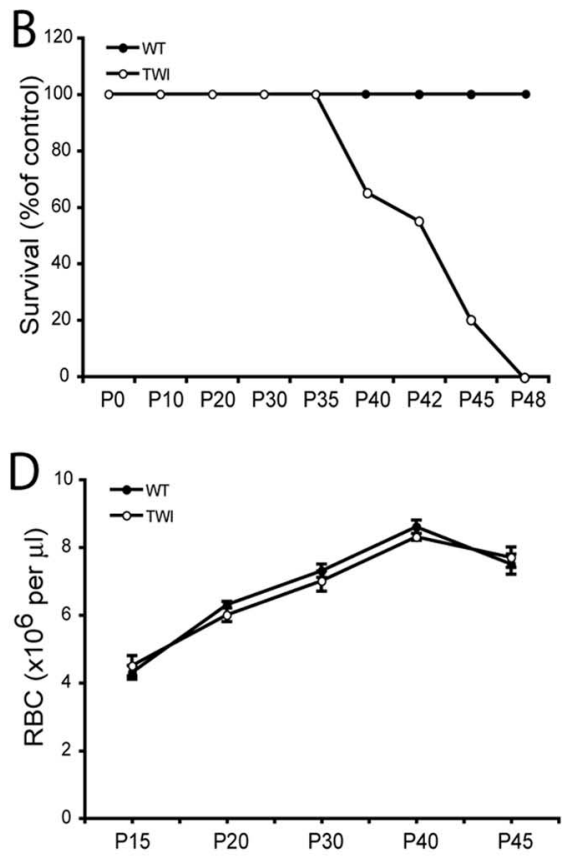

\section{Postnatal days}

Figure 1. GALC deficiency reduces circulating lymphocytes but not erythrocytes. $A, B, B$ ody weight and survival curves show that the deficiency of GALC prevents Twitcher mutant mice (TWI) to gain normal weight $(\boldsymbol{A})$ and to survive beyond $48 \mathrm{~d}(\boldsymbol{B}) . \boldsymbol{C}, \boldsymbol{D}$ Blood counts during postnatal development indicate that $T$ witcher mice develop a progressive lymphopenia $(\boldsymbol{C})$, whereas red blood counts

microscopy showed that P40 Twitcher thymus contained numerous abnormal membranous inclusions (Fig. $4 J$ ), which were not found in control samples (Fig. 4L). High-resolution electron microscopy revealed that these inclusions were multilamellar giant bodies (Fig. $4 K)$.

\section{Psychosine accumulates in GLD thymus in a nonprogressive manner}

Next, we studied the accumulation of psychosine in the thymus by mass spectrometry. Figure $5 A$ shows that thymus GALC activity was absent in the Twitcher mutant, although progressively increased in wild-type littermates over the first weeks of age. Figure 4, $B$ and $C$, shows representative LC-MS-MS chromatograms from wild-type (Fig. 5B) and Twitcher (Fig. 5C) P40 thymuses. Psychosine level was instead higher in mutant samples, accumulating to a concentration of $\sim 0.3 \mathrm{nM}$, which is approximately fivefold higher than that found in wild-type thymuses (Fig. 5D). Psychosine did not progressively accumulate in the mutant thymus, remaining between 0.2 and $0.3 \mathrm{nM}$ during the entire postnatal life.

Because psychosine has been depicted as a potent neurotoxin, we evaluated the hypothesis that this sphingolipid might contribute to death of thymocytes, causing thymic atrophy. Thymocytes were prepared from P15 wild-type and Twitcher thymuses and cultured in the presence and absence of psychosine. In culture, Twitcher thymocytes survived as well as wild-type cells (data not shown). When cultures were supplemented with psychosine, this neurotoxin only caused Twitcher and wild-type thymocytes death when its concentration reached $25 \mu \mathrm{M}$ (Fig. $5 E$ ), whereas at lower concentrations, it did not affect cell viability. Together, these results indicate that thymic involution in Twitcher mutants is associated with abnormal thymic cell death, which however cannot be explained by psychosine accumulation. 
A

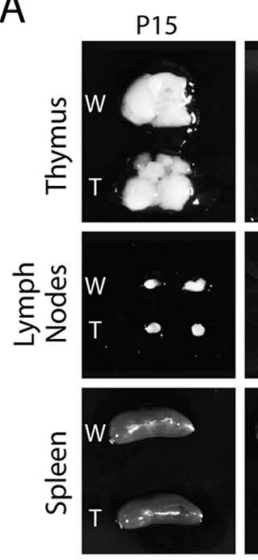

C

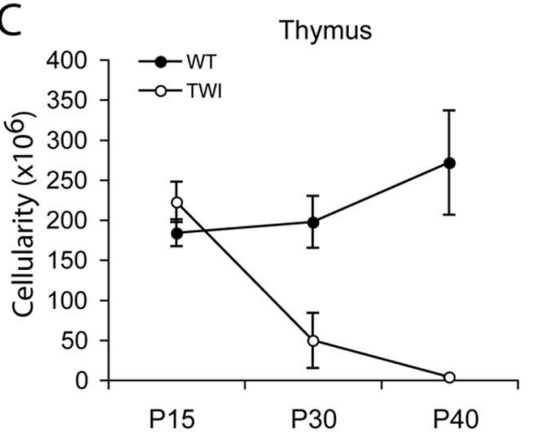

E

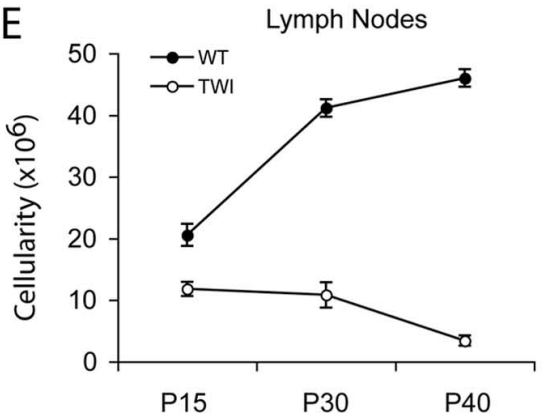

B
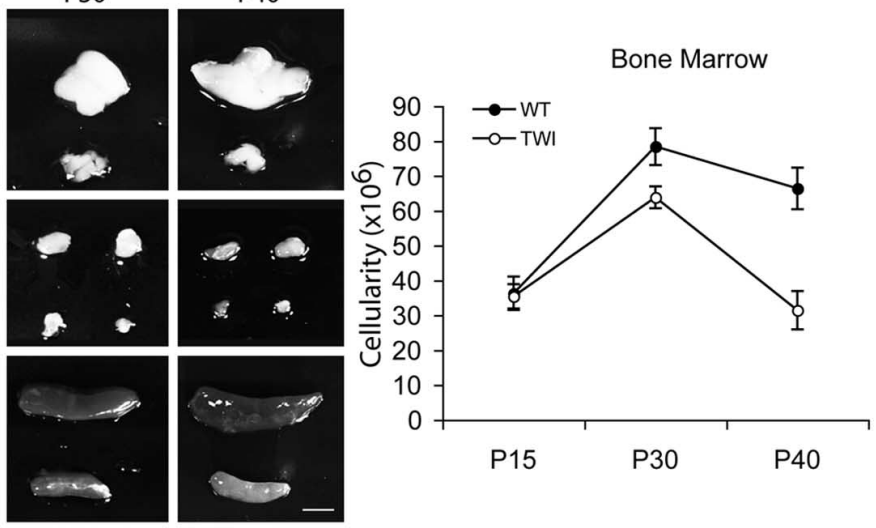

$\mathrm{D}$

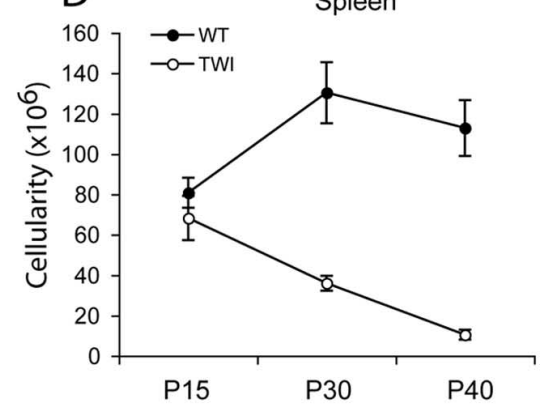

$\mathrm{F}$

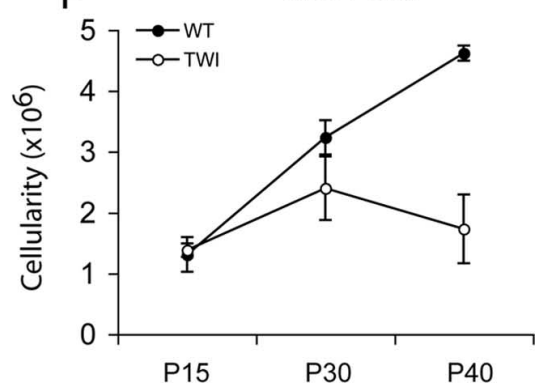

Figure 2. GALC deficiency is accompanied by atrophy of lymphoid organs. $A$, The pictures show a whole-mount comparison of primary and secondary lymphoid organs from Twitcher (T) and wild-type (W) littermate mice during postnatal age. Mutant mice showed a progressive atrophy of thymuses, lymph nodes, and spleens, which coincided in time with the appearance of neurological symptoms. $\boldsymbol{B}-\boldsymbol{F}$, Organ cellularity decreases during disease. Organ cellularity was defined by Trypan Blue negative cell counts of femurs- and tibiae-derived bone marrow cells $(\boldsymbol{B})$; axillary, mesenteric, and inguinal pooled lymph nodes $(\boldsymbol{E}) ; 500 \mu \mathrm{l}$ of peripheral blood $(\boldsymbol{F})$; and intact thymus $(\boldsymbol{C}$ ) and spleen (D). Twitcher mice (TWI) showed reduced cell numbers in the thymus, lymph nodes, and spleens between 15 and $30 \mathrm{~d}$ of age and remained lower than controls afterward. Bone marrow and blood cell numbers decreased in Twitcher mice starting from $30 \mathrm{~d}$ of age, indicating that these two compartments are affected during the late stage of the disease. Data are expressed as the mean \pm SE from three to five mice for each group per time point.

Hematopoietic stem cell transplantation improves survival of GLD mice but is unable to prevent immune atrophy

To determine whether the reduction in circulating leukocytes could be attributed to a cell autonomous defect of Twitcher hematopoietic precursors, Twitcher CD45.2 $2^{+}$bone marrow cells were transplanted into 5-d-old congenic CD45.1 ${ }^{+}$sublethally conditioned wild-type recipients. Wild-type CD $45.1^{+}$donor HSCs were also transplanted into $\mathrm{CD} 45.2^{+}$Twitcher and CD45.2 ${ }^{+}$wild-type conditioned recipients as controls. Analysis of chimerism showed that immune-reconstitution was similar, with an average engraftment of donor-derived cells of $\sim 75 \%$ (Fig. 6A).

Wild-type mice transplanted with Twitcher or wild-type HSC did not show any sign of health deterioration, suggesting that Twitcher bone marrow precursors do not transfer disease (data not shown). Furthermore, transplanted mice revealed comparable numbers of circulating WBCs and RBCs (Fig. 6B,C), suggesting that Twitcher HSC can normally develop into GALC-sufficient recipients. In contrast, Twitcher mice transplanted with wild-type HSC had a significant delay in disease progression, as expected and survived to an average of $80 \pm 3 \mathrm{~d}(n=3-5)$, they showed deterioration in their health toward the end of their lives, which included tremor, ataxia, and growing weakness (data not shown). Disease progression was again paralleled by a rapid decay of WBC after P40 (Fig. $6 \mathrm{~B}$ ). At the time of death, WBC counts in these mice were approximately threefold less than those found in the other experimental groups. In contrast, RBC numbers in transplanted Twitcher mice remained normal at any time of the study (Fig. 6C), as in the case of not-transplanted Twitcher mutants.

Reduced number of WBC correlated with thymic atrophy in transplanted Twitcher mice (Fig. 6D). DN, DP, CD4 ${ }^{+}$ $\mathrm{SP}$, and $\mathrm{CD}^{+} \mathrm{SP}$ cells were reduced fourfold, sixfold, ninefold, and sevenfold, respectively in Twitcher mice recipients of wild-type precursors compared with wild-type recipients (Fig. 6D). Similarly, the number of $\mathrm{B} 220^{+}, \mathrm{CD} 4^{+}$, $\mathrm{CD}^{+}$, and $\mathrm{CD} 11 \mathrm{~b}^{+}$cells in the spleen of Twitcher recipients was sevenfold, fourfold, threefold, and fourfold less, respectively, than those found in control recipients. These results indicate that the progressive atrophy of Twitcher lymphoid organs is mainly caused by epigenetic defects affecting thymic function rather than a cell autonomous defect of lymphoid precursors and cannot be prevented by HSC cell therapy.

Epigenetic thymic dysfunction in GLD mice is associated to a progressive loss of sympathetic fibers and deregulated expression of $\boldsymbol{\beta}$-adrenergic receptors Innervation of thymus by nonmyelinated sympathetic and parasympathetic nerves has been shown to be important for thymus function and to maintain $\mathrm{T}$ cell maturation. To examine whether thymic innervation was affected in the mutant, we analyzed thymic TH expression by confocal microscopy, the key enzyme in the synthesis of norepinephrine, which has been used previously to study axonal endings from sympathetic motorneurons in the thymic cortex (Fig. 7A-F). We found that at P7, both GLD and wild-type thymuses did not differ significantly in the density and distribution of $\mathrm{TH}^{+}$fibers (data not shown). However, at P15, GLD thymuses showed less $\mathrm{TH}^{+}$fibers within the thymic cortex (Fig. 7A) compared with age-matched wild-type tissue (Fig. 7B). P30 GLD thymuses had scarce $\mathrm{TH}^{+}$axons throughout the cortex with TH expression still detectable in some T cells (Fig. 7C,D). TH expression in the GLD thymic cortex was 
minimal at P40 (Fig. 7E). In contrast, agematched wild-type thymuses showed profuse innervation within the thymic cortex (Fig. 7F). We also studied whether cholinergic innervation (the other main innervation type in the thymus) was affected during disease. Similar to noradrenergic fiber loss, we found that the mutant thymus had reduced cholinergic fibers, detected by immunohistochemistry for ChaT (Fig. $7 G, H)$. Morphometry counting confirmed the reduction of $\mathrm{TH}+$ fibers in the cortex of GLD thymuses (Fig. 7I). At P30, the GLD thymic cortex contained $\sim 50 \%$ less fiber than the age-matched control.

Finally, we investigated whether the loss of sympathetic axons had any effect on the expression of $\beta$-adrenergic norepinephrine receptors in the mutant thymus. We quantitatively measured the expression of the receptor transcripts by realtime PCR. Figure 7J shows that expression of both receptors was drastically downregulated in the GLD thymus from early age. This weak sympathetic tone in the mutant thymus preceded organ atrophy, which occurred between P30 and P40 (Fig. 2A).

\section{Discussion}

Two new and major aspects of the pathophysiology of Krabbe disease are reported here. First, we found that GLD progression results in the atrophy of primary lymphoid organs, with the thymus and later the bone marrow being severely and irreversibly affected. This results in peripheral lymphodepletion, rendering the Twitcher mice immune-incompetent after the onset of the neurological disease. Second, we found that as GLD mice experience progressive lymphoid atrophy, autonomic innervation of these organs is reduced.

\section{GLD, a neurological disease \\ compromising immune-competence}

GLD is conventionally regarded as a neurological disease, because its cardinal feature, demyelination, is associated with a rapid progressive neurodegeneration of the nervous system. In the Twitcher mouse, a bona fide mouse model for infant variants of GLD, dysfunction of lymphoid organs was acquired progressively. By day 15 of life, the immune phenotype of control and Twitcher mutants was comparable, with thymuses and peripheral lymphoid organs of similar size, and a normal representation of immature and mature lymphocyte subsets in the mutant. However, cellularity in the mutant thymus and spleen rapidly decreased with disease onset, reaching $25-30 \%$ of control tissues by $30 \mathrm{~d}$ of age. This involution was followed by a rapid decrease in the number of mature peripheral T cells, and a reduction in cellularity of other peripheral lymphoid tissues. By the time of death, mutant mice showed complete failure to maintain normal thymic and lymphoid function, acquiring a state of immuneincompetence. These results introduce a new level of complexity in the pathophysiology of Krabbe disease where thymic function first and peripheral lymphoid organ function later, are profoundly compromised. We propose that the impairment of immune functions may contribute to worsening disease evolution in GLD individuals, weakening their ability to resist viral infections, allergies, and/or tumor formation.

\section{Cell autonomous and epigenetic mechanisms contributing to immune deficiency in GLD}

The substrates for the GLD-encoded enzyme, GalCer, and psychosine are sphingosine-rich galactolipids, which likely accumulate in membrane lipid microdomains where they exert a pleiotropic function (Simons and Ikonen, 1997; Brown and London, 1998). For instance, the clustering of GalCer within rafts facilitates bacterial and viruses such as HIV-1 initial cell adhesion (Bhat et al., 1991; Harouse et al., 1991; Fantini et al., 2000). Sphingolipids also displace cholesterol from rafts (Megha and London, 2004; Yu et al., 2005; Megha et al., 2006) altering signaling through the membrane. Thus, abnormal sphingolipid catabolism or accumulation in lipid microdomains might result in a cell autonomous defect accounting for GLD hematopoietic stem cells compromised functionality (i.e., proliferation, migration, and maturation). Furthermore, because psychosine directly deregulates some specific protein kinase $\mathrm{C}$ isozymes (Hannun and Bell, 1987), activates phospholipase A2 (Giri et al., 2006), and modifies mitochondrial membrane potential (Tapasi, 1998) and peroxisomal activity (Khan et al., 2005), its accumulation within thymic stromal tissue or in $\mathrm{T}$ cells might also directly interfere with cell survival. Of note, a recent study reported that psychosine favors proinflammatory cytokine secretion by peripheral blood mononuclear cells derived from Krabbe patients (Formichi et al., 2007; Pasqui et al., 2007). Our findings showing that lymphopoiesis but not erythropoiesis is impaired in Twitcher mutants appear to support the possibility that GALC mutation exert a diverse effect depending on the cell type. Although we found that psychosine levels were higher in the thymus of Twitcher mutants when compared with control mice as soon as P5, thymic development was preserved until P15. In addition, 
Table 1. Cell type composition in bone marrow and spleen

\begin{tabular}{|c|c|c|c|c|c|}
\hline & \multicolumn{2}{|l|}{ Bone marrow } & & \multicolumn{2}{|l|}{ Spleen } \\
\hline & Wild type & Twitcher & & Wild type & Twitcher I \\
\hline B220 & & & B220 & & \\
\hline P15 & $7.3 \pm 1.4$ & $6.5 \pm 1.5$ & P15 & $63.6 \pm 8.3$ & $49.6 \pm 7.9$ \\
\hline P30 & $8.8 \pm 1.7$ & $4.9 \pm 1.4$ & P30 & $80.2 \pm 10.0$ & $26.1 \pm 2.9$ \\
\hline P40 & $5.4 \pm 1.7$ & $0.9 \pm 0.5$ & P40 & $111.5 \pm 10.0$ & $8.2 \pm 1.3$ \\
\hline SCA-1 & & & $\mathrm{CD} 4$ & & \\
\hline P15 & $0.9 \pm 0.1$ & $0.7 \pm 0.1$ & P15 & $4.5 \pm 0.4$ & $3.5 \pm 0.4$ \\
\hline P30 & $2.3 \pm 0.2$ & $2.0 \pm 0.1$ & P30 & $20.3 \pm 3.2$ & $8.6 \pm 0.8$ \\
\hline P40 & $2.5 \pm 0.3$ & $0.8 \pm 0.3$ & P40 & $21.6 \pm 0.2$ & $3.5 \pm 1.0$ \\
\hline GR-1 & & & CD8 & & \\
\hline P15 & $2.2 \pm 0.2$ & $2.4 \pm 0.3$ & P15 & $2.3 \pm 0.3$ & $2.0 \pm 0.4$ \\
\hline P30 & $6.9 \pm 0.6$ & $6.0 \pm 0.5$ & P30 & $9.4 \pm 1.4$ & $4.5 \pm 0.3$ \\
\hline P40 & $7.3 \pm 0.9$ & $2.5 \pm 1.1$ & P40 & $9.6 \pm 0.3$ & $1.6 \pm 0.5$ \\
\hline TER-119 & & & CD11b & & \\
\hline P15 & $7.7 \pm 0.6$ & $7.8 \pm 0.1$ & P15 & $3.0 \pm 0.3$ & $2.7 \pm 0.6$ \\
\hline P30 & $19.1 \pm 1.7$ & $17.1 \pm 1.3$ & P30 & $5.5 \pm 0.3$ & $1.2 \pm 0.3$ \\
\hline P40 & $13.9 \pm 2.2$ & $11.1 \pm 1.9$ & P40 & $5.5 \pm 0.4$ & $0.2 \pm 0.1$ \\
\hline
\end{tabular}

Profile of the cellular phenotypes in bone marrow and spleen of Twitcher and wild-type mice. We used the following immunephenotypic markers: B220 for B-lymphocytes, SCA-1 for haematopoietic stem cells, GR-1 for granulocytes, Ter119 for erythroid progenitors, and CD11b for macrophages. Data are expressed as the mean \pm SE of at least three mice of each group at each time point.
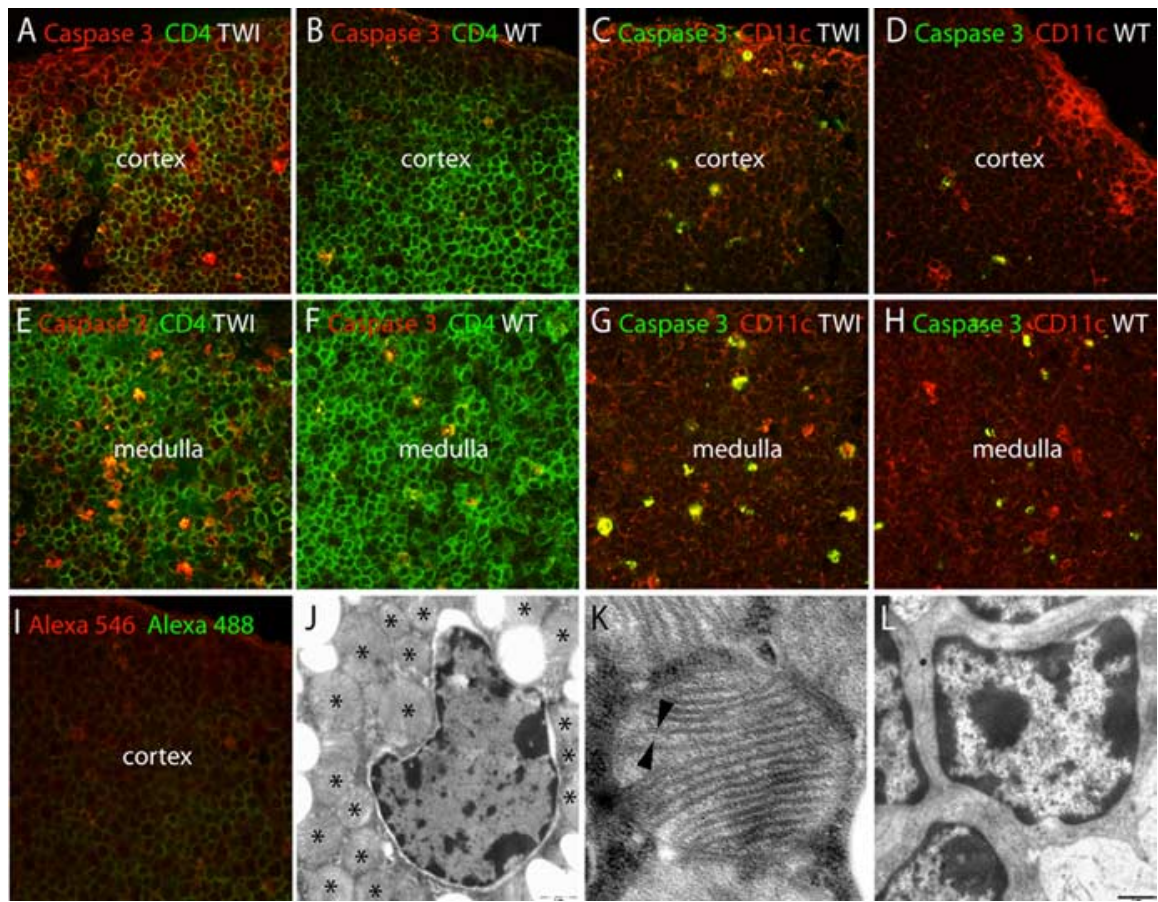

Figure 4. Apoptosis and ultrastructural changes during GLD thymic atrophy. $A-I$, Apoptotic cell death was studied by activation of caspase 3 in Twitcher (TWI) and wild-type (WT) littermates at P15 $(\boldsymbol{A}, \boldsymbol{B}, \boldsymbol{E}, \boldsymbol{F})$ and P30 (C, D, G, H). Caspase 3-positive cells (red) were numerous in CD4 positive (green) T cells in the Twitcher thymic cortex $(\boldsymbol{A})$ and medulla $(\boldsymbol{E})$, respectively, controls. Caspase 3 was also active (green) in CD11c (red) stromal cells in the Twitcher thymic cortex ( $\boldsymbol{C}$ ) and medulla $(\boldsymbol{G})$. I shows the background staining in P30 thymus. J-L, Transmission electron microscopy of ultrathin sections of Twitcher $(\boldsymbol{J}, \boldsymbol{K})$ and wild-type thymus $(\boldsymbol{L})$ showed ultrastructural changes in thymic cells with abundant multilamellar giant bodies ( $\boldsymbol{J}$, asterisks), composed of bilayered membranes ( $\boldsymbol{K}$, arrowheads). Scale bar: (in $\boldsymbol{J}, \boldsymbol{L}$ ) $1 \mu \mathrm{m}$.

psychosine-related toxicity was observed in vitro assay only at concentrations higher than those found in vivo. Thus, an increase of psychosine concentration within developing thymocytes does not appear to directly correlate with the immune phenotype of Twitcher mutants. Accordingly, transplantation of GLD HSC in normal congenic recipients allowed normal lymphopoiesis. Although it remains to be determined whether enzyme crosscorrection by host cells mitigate the GALC deficiency of donor Twitcher cells (Neufeld and Fratantoni, 1970; Kondo et al., 2005; Givogri et al., 2006), the finding that GLD recipients of normal congenic HSC became progressively lymphopenic, is rather indicative of the existence of epigenetic factors contributing to thymic involution in GLD. Although we do not propose lymphoid atrophy as causative for GLD, we believe it is an additive pathological factor contributing to a very complex and rapidly degenerating phenotype.

\section{Autonomic denervation of the GLD thymus}

We found that loss of autonomic tone precedes and possibly accounts for the initiation of thymic involution. The nervous system exerts a significant influence on immune organs by direct innervation (Besedovsky and Sorkin, 1977; Elenkov et al., 2000; Wrona, 2006) and by the action of hormones such as somatotrophin (Savino et al., 2002). Primary (bone marrow and thymus) and secondary (lymph nodes and spleen) lymphoid organs are highly innervated by nonmyelinated sympathetic projections from central motor neurons and release neurotransmitters, including acetylcholine (Tominaga et al., 1992; Rinner et al., 1999). Most of sympathetic axons in the thymus originate from postganglionic neurons located in the superior cervical and stellate ganglia (Bulloch and Pomerantz, 1984; Nance et al., 1987). Tracing analysis indicates that thymic sympathetic outflow directly starts in neurons from the thoracic spinal cord, brainstem, caudal raphe nucleus, and hypothalamus (Trotter et al., 2007). Furthermore, the neurotransmitter norepinephrine (Singh and Owen, 1976; Felten et al., 1987; Morale et al., 1992; Tsao et al., 1996; Kurz et al., 1997; von Patay et al., $1998,1999)$ is found in the thymus, and thymic cells express both $\beta 1$ - and $\beta 2$-adrenergic receptors (Morale et al., 1992; Kurz et al., 1997; von Patay et al. 1999) able to modulate maturation of T precursor cells and influence the thymic microenvironment. 
A

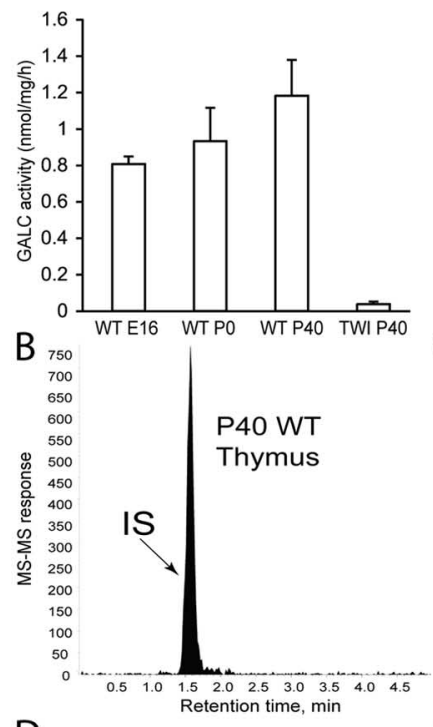

$\mathrm{D}$

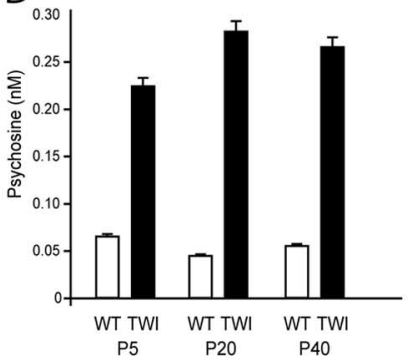

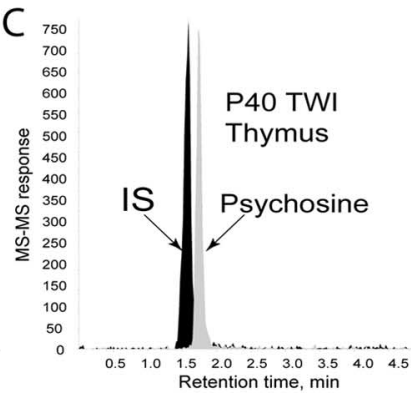

E

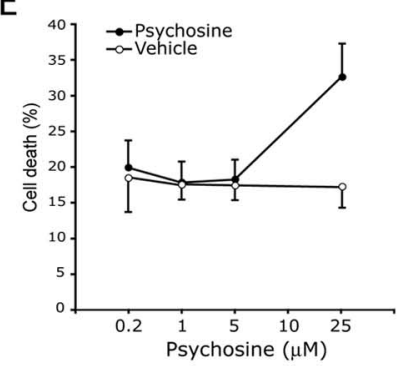

Figure 5. Determination of psychosine in GLD thymus. $\boldsymbol{A}$, The GLD thymus lacks GALC activity. The enzyme was active in wild-type (WT) thymus from embryonic day 16 (E16) to P40. Data are expressed as the mean \pm SE from three thymuses per time point. $\boldsymbol{B}, \boldsymbol{C}$, LC-MS-MS chromatograms showing the absence of psychosine in wild-type thymus $(\boldsymbol{B})$ and the presence of the neurotoxin in Twitcher thymus (C). Lactosyl-sphingosine was used as an internal standard (IS). D, Quantification of psychosine during the postnatal development of the wild-type and Twitcher thymuses. Data are expressed as the mean \pm SEfrom two to three thymuses for each time point per genotype. $\boldsymbol{E}$, The effect of psychosine on thymocytes survival was studied in vitro using wild-type thymocytes from P15 mice. Cultures were exposed to different amounts of the sphingolipid for $1 \mathrm{~d}$ before mitochondrial membrane potential was determined as described. Data are expressed as the mean percentage of cell death \pm SE from two cultures per dose.

Thus, a link between the brain and the thymus is supported at different levels. We found that the GLD thymus presents a progressive and irreversible depletion of cathecolaminergic $\left(\mathrm{TH}^{+}\right)$ and cholinergic $\left(\mathrm{ChaT}^{+}\right)$axons, comprising fibers innervating the thymic cortex and medulla, detectable as early as P15 and complete toward the fifth week of life. Thymic denervation was accompanied by reduced expression of both $\beta 1$ - and $\beta 2$ adrenergic receptors and increased apoptosis, suggesting that sympathetic tone participates in maintaining normal expression of adrenergic receptors. The early and progressive loss of sympathetic tone in GLD lymphoid organs likely accounts for thymic involution and for the failure of normal congeneic bone marrow cells to reconstitute Twitcher thymic function. By the time donor cells entered in the organ, the GLD thymus innervation was already compromised, possibly resulting in a microenvironment unable to support $\mathrm{T}$ cell maturation.

The relevance of sympathetic tone on the function of the thymus has been speculated for several years. Atrophy of thymus and spleen has been observed in various other diseases, which cause neurological degeneration. For example, mouse models of motor neuron disease like the wasted mouse (Kaiserlian et al., 1986) and the MND2
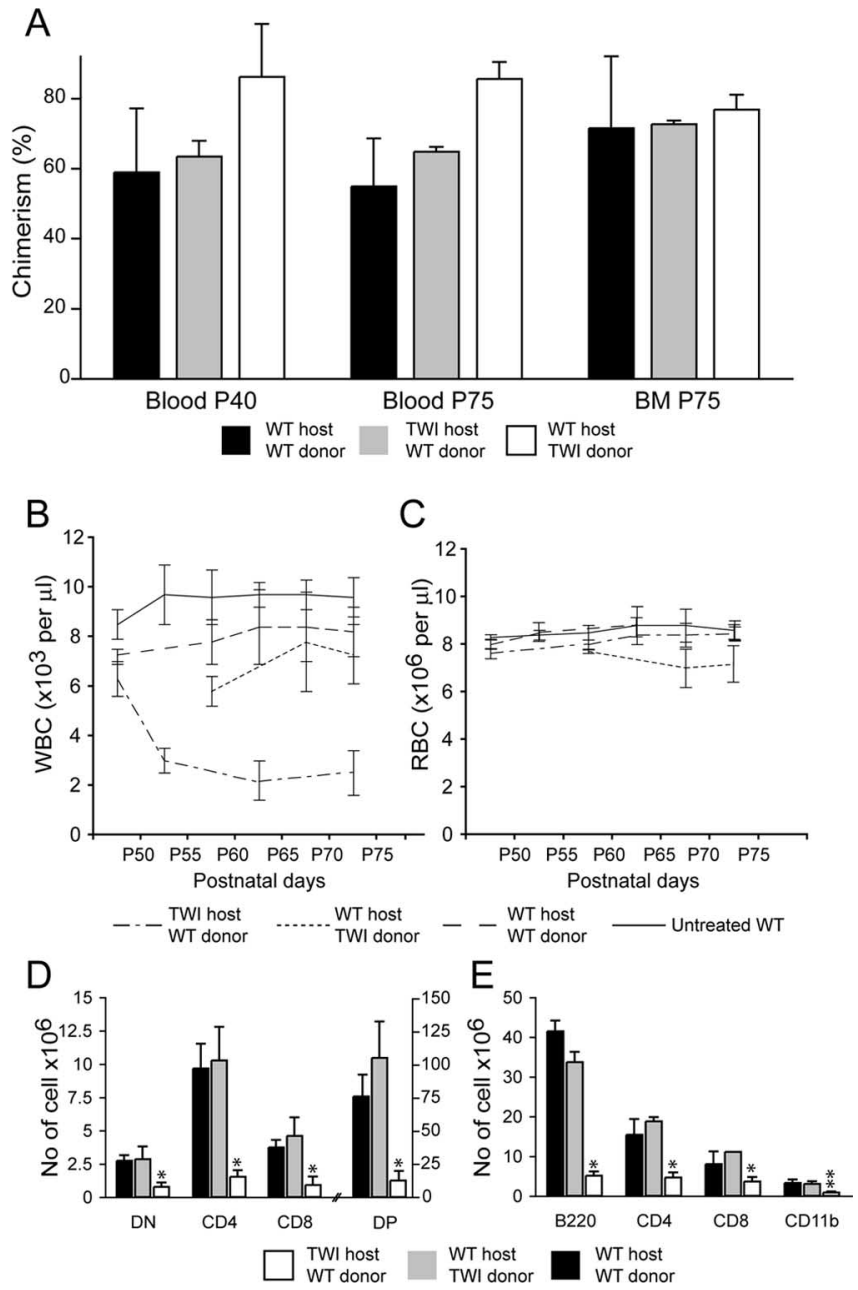

Figure 6. Hematopoietic stem cell transplantation supports the existence of an epigenetic defect hampering thymic development in GLD mice. $\boldsymbol{A}$, Transplantation of bone marrowderived cells was performed between Twitcher (TWI) and wild-type congenic mice to test for epigenetic defects. Donor cell engraftment was measured in the blood at P40 and P75 and in the bone marrow (BM) at P75. Chimerism was similar in each of the three groups of transplanted mice, with an average engraftment of $\sim 75 \%$. B, C, WBC counts were comparable in wild-type mice transplanted with either wild-type or Twitcher bone marrow cells but reduced in Twitcher mice transplanted with wild-type bone marrow. This suggests that the lymphopoiesis in the Twitcher mutant is affected by a noncell autonomous defect. $\boldsymbol{D}, \boldsymbol{E}$, Cellular composition of thymus $(\boldsymbol{D})$ and spleen $(\boldsymbol{E})$ after BM transplant. Normal numbers and distribution of thymocytes subsets were observed in mice that received wild-type or Twitcher bone marrow cells. In contrast, transplanted Twitcher mice showed a severe reduction in the number of DN, CD4, CD8, and DP thymic cells (D) and B220, CD4, CD8, and CD11b (E) splenocytes. Data are expressed as the mean \pm SE from three to five mice for each group. ${ }^{*} p<0.01 ;{ }^{* *} p<0.05$ versus wild-type recipient of wild-type transplanted cells.

mouse (Jones et al., 1993), which develop dysfunction of spinal motor neurons and of cerebellar dysfunction and ataxia, like the staggerer mouse (Trenkner and Hoffmann, 1986), and models of experimental axonal loss (hemilateral vagotomy and administration of guanethidine) (Kendall and Al Shawaf, 1991; Antonica et al., 1996; Gulati et al., 1999), reveal progressive immunodeficiency resulting from hypoplasia and abnormal maturation of lymphoid organs. Although a review of the published bibliography and consultation with pediatric neurologists failed to provide observation on thymic development in newborn children with Krabbe disease before and after disease onset, children affected by spinal muscular atrophy (Ryniewicz and Pawinska, 1978) or by ataxia-telangiectasia (Peterson et al., 1964) show evidence of lymphoid organs atrophy and impaired cellular immunity. 

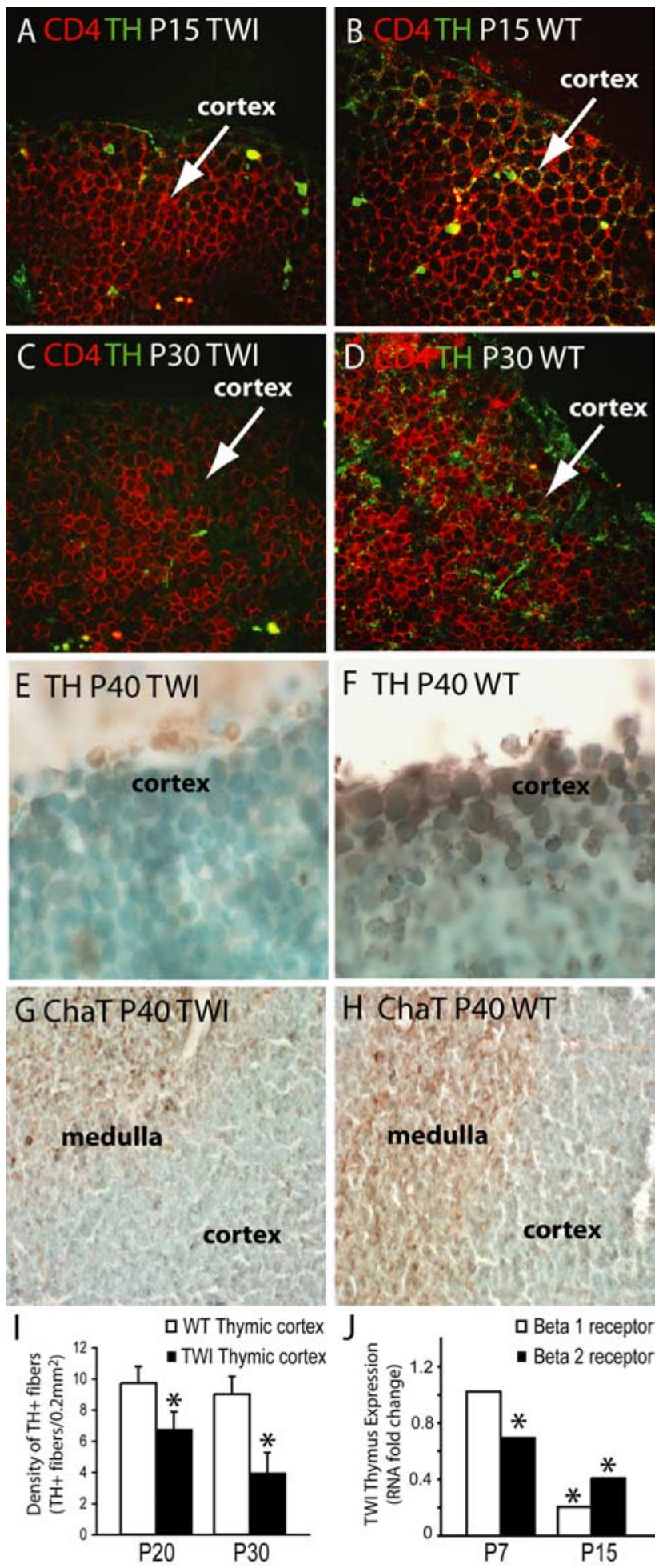

Figure 7. Progressive axonal loss from autonomic fibers in the atrophied GLD thymus. $\boldsymbol{A}-\boldsymbol{D}$ Confocal immunohistochemistry of $\mathrm{TH}$ (green) showed reduced innervation of norepinephrine fibers in the Twitcher (TWI) cortex at P15 ( $\boldsymbol{A})$ and P30 ( $\boldsymbol{C}$, when compared with age-matched controls $(\boldsymbol{B}, \boldsymbol{D})$. A CD4 counterstaining (red) was used. $\boldsymbol{E}-\boldsymbol{H}$, Light microscopy immunohistochemistry for TH $(\boldsymbol{E}, \boldsymbol{F})$ and $\mathrm{ChaT}(\boldsymbol{G}, \boldsymbol{H})$ in P40 thymuses showed reduced expression of TH (E) and $C$ haT $(\boldsymbol{G})$ in the aged TWI thymus. $I$, The density of TH + fibers was quantified and expressed as the number of $\mathrm{TH}+$ fibers per $0.2 \mathrm{~mm}^{2}$ of thymic cortex at P20 and P30. Data averages two independent experiments. $p<0.05$ versus wild type. J, Fold changes in expression of norepinephrine receptors ( $\beta 1$ and $\beta 2$ ) was quantified by real-time PCR in RNA isolated from thymuses at P7 and P15. Data are normalized to the expression of the GAPDH housekeeping gene and compared with values obtained in age-matched wild-type tissues (the expression of which equals a value of 1). Data average two independent experiments. $p<0.05$ versus wild type.
Degeneration of thymus and lymphoid tissue in GLD has not been investigated previously, possibly because GLD has always been considered a myelin disorder. Although not addressed in this study, in a parallel investigation, we also found evidence of a cell autonomous defect affecting several types of neurons along the neuroaxis of the GLD model (M. I. Givogri and E. R. Bongarzone, unpublished observations. Because autonomic axons are mostly unmyelinated, our results provide evidence that axonal neuropathy in GLD might also occur independent from demyelination. Together, these findings indicate a crucial role for axonal function in the regulation of various visceral functions, including thymus and secondary lymphoid organs and also at the base for other diseases such as diabetes (Razavi et al., 2006). At this time, we cannot exclude that both epigenetic (loss of autonomic innervation of lymphoid organ) and cell autonomous (GALC deficiency in hematopoietic precursors) contribute to disease progression. Better defining this issue will be critical for the development of appropriate therapeutic options.

\section{References}

Antonica A, Ayroldi, E, Magni F, Paolocci N (1996) Lymphocyte traffic changes induced by monolateral vagal denervation in mouse thymus and peripheral lymphoid organs. J Neuroimmunol 64:115-122.

Besedovsky H, Sorkin E (1977) Network of immune-neuroendocrine interactions. Clin Exp Immunol 27:1-12.

Bhat S, Spitalnik SL, Gonzalez-Scarano F, Silberberg DH (1991) Galactosyl ceramide or a derivative is an essential component of the neural receptor for human immunodeficiency virus type 1 envelope glycoprotein gp120. Proc Natl Acad Sci USA 88:7131-7134.

Brown DA, London E (1998) Functions of lipid rafts in biological membranes. Annu Rev Cell Dev Biol 14:111-136.

Bullard W, Southard E (1906) Difuse gliosis of the cerebral white matter in a child. J Nerv Ment Dis 33 188-193.

Bulloch K, Pomerantz W (1984) Autonomic nervous system innervation of thymic-related lymphoid tissue in wildtype and nude mice. J Comp Neurol 228:57-68.

Crome L, Hanefeld F, Patrick D, Wilson J (1973) Late onset globoid cell leucodystrophy. Brain 96:841-848.

Dolcetta D, Perani L, Givogri MI, Galbiati F, Amadio S, Del Carro U, Finocchiaro G, Fanzani A, Marchesini S, Naldini L, Roncarolo MG, Bongarzone E (2006) Design and optimization of lentiviral vectors for transfer of GALC expression in Twitcher brain. J Gene Med 8:962-971.

Elenkov IJ, Wilder RL, Chrousos GP, Vizi ES (2000) The sympathetic nerve-an integrative interface between two supersystems: the brain and the immune system. Pharmacol Rev 52:595-638.

Escolar ML, Poe MD, Provenzale JM, Richards KC, Allison J, Wood S, Wenger DA, Pietryga D, , Champagne M, Morse R, Krivit W, Kurtzberg J (2005) Transplantation of umbilical-cord blood in babies with infantile Krabbe's disease. N Engl J Med 352:2069-2081.

Fantini J, Maresca M, Hammache D, Yahi N, Delézay O (2000) Glycosphingolipids (GSL) microdomain as attachment platforms for host pathogens and their toxins on intestinal epithelial cells: activation of signal transduction pathways and perturbations of intestinal absorption and secretion. Glycoconj J 17:173-179.

Felten DL, Felten SY, Bellinger DL, Carlson SL, Ackerman KD, Madden KS, Olschowki JA, Livnat S (1987) Noradrenergic sympathetic neural interactions with the immune system: structure and function. Immunol Rev 100:225-260.

Formichi P, Radi E, Battisti C, Pasqui A, Pompella G, Lazzerini PE, LaghiPasini F, Leonini A, Di Stefano A, Federico A (2007) Psychosineinduced apoptosis and cytokine activation in immune peripheral cells of Krabbe patients. J Cell Physiol 212:737-743.

Giri S, Khan M, Rattan R, Singh I, Singh AK (2006) Krabbe disease: psychosine-mediated activation of phospholipase A2 in oligodendrocyte cell death. Lipid Res 47:1478-1492.

Givogri MI, Costa RM, Schonmann V, Silva AJ, Campagnoni AT, Bongarzone ER (2002) Central nervous system myelination in mice with deficient expression of Notch1 receptor. J Neurosci Res 67:309-320.

Givogri MI, Galbiati F, Fasano S, Amadio S, Perani L, Superchi D, Morana P, Del Carro U, Marchesini S, Brambilla R, Wrabetz L, Bongarzone E (2006) Oli- 
godendroglial progenitor cell therapy limits central neurological deficits in mice with metachromatic leukodystrophy. J Neurosci 26:3109-3119.

Gulati P, Tay S, Leong SK (1999) Ultrastructural changes in the chick thymus following unilateral vagomoty. Anat Rec 255:261-270.

Hagberg B, Kollberg H, Sourander P, Akesson HO (1970) Infantile globoid cell leukodystrophy (Krabbe's disease): a clinical and genetic study of 32 Swedish cases 1953-1967. Neuropaediatrie 1:74-88.

Hannun YA, Bell RM (1987) Lysosphingolipids inhibit protein kinase C: implications for thesphingolipidoses. Science 235:670-674.

Harouse JM, Bhat S, Spitalnik SL, Laughlin M, Stefano K, Silberberg DH, Gonzalezscarano F (1991) Inhibition of entry of HIV-1 in neural celllines by antibodies against galactosyl ceramide. Science 253:320-323.

Hoogerbrugge PM, Poorthuis BJ, Wagemaker G, van Bekkum DW, Suzuki K (1989) Alleviation of neurologic symptoms after bone marrow transplantation in twitcher mice. Transplant Proc 21:2980-2981.

Jones JM, Albin RL, Feldman EL, Simin K, Schuster TG, Dunnick WA, Collins JT, Chrisp CE, Taylor BA, Meisler MH (1993) mnd2: a new mouse model of inherited motor neuron disease. Genomics 16:669-677.

Kaiserlian D, Savino W, Uriel J, Hassid J, Dardenne M, Bach JF (1986) The wasted mutant mouse. II. Immunological abnormalities in a mouse described as a model of ataxia-telangiectasia. Clin Exp Immunol 63:562-569.

Kendall MD, Al Shawaf A (1991) Innervation of the rat thymus gland. Brain Behav Immun 5:9-28.

Khan M, Haq E, Giri S, Singh I, Singh AK (2005) Peroxisomal participation in psychosinemediated toxicity: implications for Krabbe's disease. J Neurosci Res 80:845.

Kondo Y, Wenger DA, Gallo V, Duncan ID (2005) Galactocerebrosidase-deficient oligodendrocytes maintain stable central myelin by exogenous replacement of the missing enzyme in mice. Proc Natl Acad Sci USA 102:18670-18675.

Krivit W, Lockman LA, Watkins PA, Hirsch J, Shapiro EG (1995) The future for treatment by bone marrow transplantation for adrenoleukodystrophy, metachromatic leukodystrophy, globoid cell leukodystrophy and Hurler syndrome. J Inherit Metab Dis 18:398-412.

Krivit W, Shapiro EG, Peters C, Wagner JE, Cornu G, Kurtzberg J, Wenger DA, Kolodny EH, Vanier MT, Loes DJ, Dusenbery K, Lockman LA (1998) Hematopoietic stem-cell transplantation in globoid-cell leukodystrophy. N Engl J Med 338:1119-1126.

Kurz B, Feindt J, von Gaudecker B, Kranz A, Loppnow H, Mentlein R (1997) Beta-adrenoceptor-mediated effects in rat cultured thymic epithelial cells. Br J Pharmacol 120:1401-1408.

Livak KL, Schmittgen TS (2001) Analysis of relative gene expression data using RealTime quantitative PCR and the 2 CT method. Methods 25:402-408.

Luddi A, Volterrani M, Strazza M, Smorlesi A, Rafi MA, Datto J, Wenger DA, Costantino-Ceccarini E (2001) Retrovirus-mediated gene transfer and galactocerebrosidase uptake into twitcher glial cells results in appropriate localization and phenotype correction. Neurobiol Dis 8:600-610.

Luzi P, Rafi MA, Zaka M, Rao HZ, Curtis M, Vanier MT, Wenger DA (2005) Biochemical and pathological evaluation of long-lived mice with globoid cell leukodystrophy after bone marrow transplantation. Mol Genet Metab 86:150-159.

Megha, London, E (2004) Ceramide selectively displaces cholesterol from ordered lipid domains (rafts): implications for lipid raft structure and function. J Biol Chem 279:9997-10004.

Megha, Bakht O, London E (2006) Cholesterol precursors stabilize ordinary and ceramide-rich ordered lipid domains (lipid rafts) to different degrees. Implications for the Bloch hypothesis and sterol biosynthesis disorders. J Biol Chem 281:21903-21913.

Morale MC, Gallo F, Batticane N, Marchetti B (1992) The immune response evokes up- and down-modulation of beta 2-adrenergic receptor messenger RNA concentration in the male rat thymus. Mol Endocrinol 6:1513-1524.

Nance DM, Hopkins DA, Bieger D (1987) Re-investigation of the innervation of the thymus gland in mice and rats. Brain Behav Immun 1:134-147.

Neufeld EF, Fratantoni JC (1970) Inborn errors of mucopolysaccharide metabolism. Science 169:141-146.

Pasqui AL, Di Renzo M, Auteri A, Federico G, Puccetti L (2007) Increased TNF-alpha production by peripheral blood mononuclear cells in patients with Krabbe's disease: effect of psychosine. Eur J Clin Invest 37:742-745.

Peterson RD, Kelly WD, Good RA (1964) Ataxia-telangiactasia. Its association with a defective thymus, immunological-deficiency disease and malignancy. Lancet 30:1189-1193.
Razavi R, Chan Y, Afifiyan FN, Liu XJ, Wan X, Yantha J, Tsui H, Tang L, Tsai S, Santamaria P, Driver JP, Serreze D, Salter MW, Dosch HM (2006) TRPV1+ sensory neurons control beta cell stress and islet inflammation in autoimmune diabetes. Cell 127:1123-1135.

Rinner I, Globerson A, Kawashima K, Korsatko W, Schauenstein K (1999) A possible role for acetylcholine in the dialogue between thymocytes and thymic stroma. Neuroimmunomodulation 6:51-55.

Ryniewicz B, Pawinska M (1978) Preliminary immunological studies in spinal muscular atrophy. Eur J Pediatr 128:57-60.

Sakai N, Inui K, Tatsumi N, Fukushima H, Nishigaki T, Taniike M, Nishimoto J, Tsukamoto H, Yanagihara I, Ozono K, Okada S (1996) Molecular cloning and expression of cDNA for murine galactocerebrosidase and mutation analysis of the twitcher mouse, a model of Krabbe's disease. J Neurochem 66:1118-1124.

Savino W, Postel-Vinay MC, Smaniotto S, Dardenne M (2002) The thymus gland: a target organ for growth hormone. Scand J Immunol 55:442-452.

Simons K, Ikonen E (1997) Functional rafts in cell membranes. Nature 387:569-572.

Singh U, Owen JJ (1976) Studies on the maturation of thymus stem cells. The effects of catecholamines, histamine and peptide hormones on the expression of T cell alloantigens. Eur J Immunol 6:59-62.

Suzuki K (2003) Evolving perspective of the pathogenesis of globoid cell leukodystrophy (Krabbe disease). Proc Jpn Acad Ser B 79:1-8.

Tapasi (1998) Effect of psychosine on mitochondrial function. Indian J Biochem Biophys 35:161-165.

Tominaga T, Fukata J, Hayashi Y, Satoh Y, Fuse N, Segawa H, Ebisui O, Nakai Y, Osamura Y, Imura H (1992) Distribution and characterization of immunoreactive corticostatin in the hypothalamic-pituitary-adrenal axis. Endocrinology 130:1593-1598.

Trenkner E, Hoffmann MK (1986) Defective development of the thymus and immunological abnormalities in the neurological mouse mutation "staggerer." J Neurosci 6:1733-1737.

Trotter RN, Stornetta RL, Guyenet PG, Roberts MR (2007) Transneuronal mapping of the CNS network controlling sympathetic outflow to the rat thymus. Auton Neurosci 131:9-20.

Tsao CW, Cheng JT, Shen CL, Lin YS (1996) 6-Hydroxydopamine induces thymocyte apoptosis in mice. J Neuroimmunol 65:91-95.

Umezawa F, Eto Y, Tokoro T, Ito F, Maekawa K (1985) Enzyme replacement with liposomes containing beta-galactosidase from Charonialumpas in murine globoid cell leukodystrophy (twitcher). Biochem Biophys Res Commun 127:663-667.

von Patay B, Loppnow H, Feindt J, Kurz B, Mentlein R (1998) Catecholamines and lipopolysaccharide synergistically induce the release of interleukin-6 from thymic epithelial cells. J Neuroimmunol 86:182-189.

von Patay B, Kurz B, Mentlein R (1999) Effect of transmitters and cotransmitters of the sympathetic nervous system on interleukin- 6 synthesis in thymic epithelial cells. Neuroimmunomodulation 6:45-50.

Wenger DA, Suzuki K, Suzuki Y, Suzuki K (2001) In the metabolic and molecular bases of inherited disease (Scriver CR, Beaudet AL, Sly WS, Valle D, Childs B, Kinzler KW, Vogelstein B, eds), pp 3669, 3670, 3687. New York: McGraw-Hill.

Wrona D (2006) Neural-immune interactions: an integrative view of the bidirectional relationship between the brain and immune systems. J Neuroimmunol 172:38-58.

Wu YP, McMahon E, Kraine MR, Tisch R, Meyers A, Frelinger J, Matsushima GK, Suzuki K (2000) Distribution and characterization of GFP(+) donor hematogenous cells in Twitcher mice after bone marrow transplantation. Am J Pathol 156:1849-1854.

Wu YP, McMahon EJ, Matsuda J, Suzuki K, Matsushima GK, Suzuki K (2001) Expression of immune-related molecules is downregulated in twitcher mice following bone marrow transplantation. J Neuropathol Exp Neurol 60:1062-1074.

Yagi T, McMahon EJ, Takikita S, Mohri I, Matsushima GK, Suzuki K (2004) Fate of donor hematopoietic cells in demyelinating mutant mouse, twitcher, following transplantation of GFP+ bone marrow cells. Neurobiol Dis 16:98-109.

Yu C, Alterman M, Dobrowsky RT (2005) Ceramide displaces cholesterol from lipid rafts and decreases the association of the cholesterol binding protein caveolin-1. J Lipid Res 46:1678-1691. 